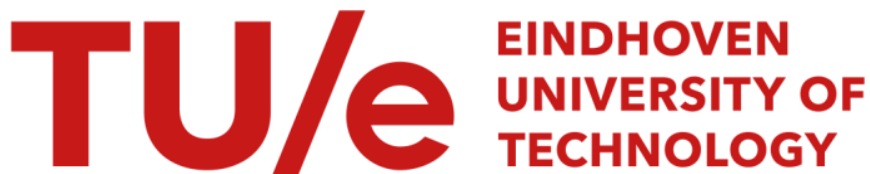

\section{3-D scene reconstruction from image sequences}

Citation for published version (APA):

Nguyen, H. V., \& Hanajik, M. (1995). 3-D scene reconstruction from image sequences. In V. Hlavac, \& R. Sara (Eds.), CAIP : computer analysis of images and patterns : international conference : proceedings, 6th, Prague, Czech Republic, September 6-8, 1995 (pp. 182-189). (Lecture Notes in Computer Science; Vol. 970). Springer.

Document status and date:

Published: 01/01/1995

\section{Document Version:}

Publisher's PDF, also known as Version of Record (includes final page, issue and volume numbers)

\section{Please check the document version of this publication:}

- A submitted manuscript is the version of the article upon submission and before peer-review. There can be important differences between the submitted version and the official published version of record. People interested in the research are advised to contact the author for the final version of the publication, or visit the $\mathrm{DOI}$ to the publisher's website.

- The final author version and the galley proof are versions of the publication after peer review.

- The final published version features the final layout of the paper including the volume, issue and page numbers.

Link to publication

\section{General rights}

Copyright and moral rights for the publications made accessible in the public portal are retained by the authors and/or other copyright owners and it is a condition of accessing publications that users recognise and abide by the legal requirements associated with these rights.

- Users may download and print one copy of any publication from the public portal for the purpose of private study or research.

- You may not further distribute the material or use it for any profit-making activity or commercial gain

- You may freely distribute the URL identifying the publication in the public portal.

If the publication is distributed under the terms of Article 25fa of the Dutch Copyright Act, indicated by the "Taverne" license above, please follow below link for the End User Agreement:

www.tue.nl/taverne

Take down policy

If you believe that this document breaches copyright please contact us at:

openaccess@tue.nl

providing details and we will investigate your claim. 


\title{
3-D Scene Reconstruction from Image Sequences
}

\author{
H. V. Nguyen and M. Hanajík \\ Eindhoven University of Technology \\ Department of Electrical Engineering, \\ Measurement and Control Section, \\ P.O.Box 513, NL-5600 MB Eindhoven, The Netherlands \\ e-mail: M.Hanajik@ele.tue.nl \\ fax: +3140 434582, tel.: +31 40473307
}

\begin{abstract}
In this paper we describe a technique for reconstruction of a static 3-D scene from a monocular image sequence. The problem is formulated as a stochastic filtering problem where state variables describe the scene and the camera parameters, and images are observations of this state. This rather general problem formulation allows us to drop requirements otherwise requested, like knowledge of the camera motion path, or the necessity to have a binocular or a trinocular image sequence. The scene is represented as a set of 3-D line segments, edges contours etc. The computation time of each state update is linear with the number of scene line segments. In the paper the achieved results are given and conclusions are derived.
\end{abstract}

\section{Introduction}

Reconstruction of a three-dimensional (3-D) scene from a sequence of images known as structure from motion has been a topic of active research in the field of computer vision in recent years. We present a technique for a static 3-D scene reconstruction by processing a sequence of monocular images. Furthermore, the knowledge of the camera motion path is not required for the scene reconstruction. The camera path is computed as a side result by the presented technique. However, some known (reference) part of the scene must be contained in a few initial images of the sequence.

The scene reconstruction is provided by a nonlinear stochastic filter. The state of the filter consists of the 3-D scene description parameters and the parameters specifying camera position and orientation. At each time instant when an image is captured and processed, the state estimate is updated, this is called a measurement update. Between the measurement updates, the state estimate is updated by system dynamics (basically camera motion dynamics), this is called a time update.

To provide correct data for filtering, correspondences between line segments in subsequent images have to be established. This is done by tracking line segments in the two-dimensional (2-D) image plane. Predictions of line segments in the next image are computed by Kalman filters, one separate filter for each line 
segment. This is an another layer of stochastic filtering. Line correspondence is found using stochastic data association.

The paper is organized as follows. In section 2 we give a brief survey of related work. In section 3 we describe the proposed technique. In subsections 3.2 , $3.3,3.4,3.5$ and 3.6 , issues concerning measurement update, time update, state initialization, line segments tracking and efficiency of computations, respectively, are treated. In section 4, our current implementation is described and achieved results are given. Finally, the conclusions are derived and further research is proposed in section 5 .

\section{Related work}

Many authors use a binocular or trinocular vision system on a mobile robot to build a local map of the environment [6], [1]. The 3-D scene is reconstructed by triangulation of the corresponding line segments from stereo matching between the three-view images. A global map of the environment is obtained by integrating a sequence of stereo frames taken, while the robot navigates in the environment [10]. Correspondences between 3-D line segments in consecutive frames are kept by tracking 2-D line segments in an image sequence of one of the cameras [5]. The displacement of the robot can be estimated given correspondences of 3-D line segments between frames, which can be used to calibrate the cameras [11]. By merging line segments observed in different frames a better estimate of the line segments is achieved.

Instead of estimating the displacement of the robot, the authors of [8] and [7] have chosen to measure its movement by additional sensors. They match feature points in binocular images by searching on corresponding epipolar lines in the other image. New observations due to camera movement will reduce the spatial uncertainty of the 3 -D points by means of information integration.

The problem of stereo matching can be avoided by tracking 2-D line segments in subsequent images. In [3], [4], [5] line segments in subsequent images are tracked, by modeling their movement. The correspondence is used to reconstruct 3-D line segments for each pair of subsequent images. The camera position is provided by a robot controller. By fusion of 3-D data from different view points, an accurate and complete representation of the scene can be obtained.

\section{Scene reconstruction by stochastic filtering}

\subsection{Representation}

We have chosen to represent a 3-D scene by a set of line segments in 3-D space. Each line segment $l$ is specified by coordinates of its begin- and endpoint in a world coordinate system, $l=\left(x_{b}, y_{b}, z_{b}, x_{e}, y_{e}, z_{e}\right)^{t}$. The camera position (and orientation) in 3 -D space is specified by six parameters $\theta=\left(\alpha, \beta, \gamma, t_{x}, t_{y}, t_{z}\right)^{t}$. A 
point with homogeneous ${ }^{1}$ coordinates $X_{w}=\left(x_{w}, y_{w}, z_{w}, 1\right)^{t}$ can be transformed into a camera centered coordinate system (coordinates $\left.X_{c}=\left(x_{c}, y_{e}, z_{c}, 1\right)^{t}\right)$ by matrix left multiplication with a $4 \times 4$ transformation matrix $M$. Obviously, matrix $M$ is a function of camera parameters $\theta=\left(\alpha, \beta, \gamma, t_{x}, t_{y}, t_{z}\right)^{t}$. Hence,

$$
X_{c}=M(\theta) X_{w} .
$$

A point with coordinates $\left(x_{c}, y_{c}, z_{c}\right)$ in the camera centered coordinate system is projected onto the image plane at coordinates $(u, v)$ given by

$$
u=f \frac{x_{c}}{z_{c}}, \quad v=f \frac{y_{c}}{z_{c}} .
$$

A line segment on the image plane is represented by the image plane coordinates of its begin- and endpoint, $q=\left(u_{b}, v_{b}, u_{e}, v_{e}\right)^{t}$. From (1) and (2) it follows that vector $q$ is a function of parameters $\theta$ and $l$ specifying the camera and 3 -D line segment positions.

We introduce a state vector $x_{t}$ specifying the 3 -D scene and the camera position at time $t$, defined as

$$
x_{t}=\left(l_{1, t}, l_{2, t} \ldots l_{n, t}, \theta_{p, t}, \theta_{c, t}\right)^{t},
$$

where $l_{i, t}, i=1 \ldots n$ specify 3 -D line segments observed so far, and $\theta_{p, t}, \theta_{c, t}$ are camera parameters at the previous time instant and the current time instant respectively.

We define an observation vector as the vector of image plane coordinates of observed line segments as

$$
y_{t}=\left(y_{c, t}, y_{p, t}\right)^{t}=\left(q_{c_{n, t}}, \ldots, q_{c_{1, t}}, q_{p_{n, t}}, \ldots, q_{p_{n-m+1, t}}\right)^{t}, 0 \leq m \leq n .
$$

Coordinates of line segments which have been observed for the first time in the current image are excluded from the observation vector and their processing is deferred until the next image. Lines $l_{1}, \ldots, l_{n-m}$ are observed for at least two times in the past and lines $l_{n-m+1}, \ldots, l_{n}$ are observed for the first time in two consecutive images (thus their processing has been deferred until now).

The state vector $x_{t}$ is unknown, and we may consider it to be an outcome of a random variable $X_{t}$. Probability distribution of $X_{t}$ expresses our knowledge of the scene and the camera position at time $t$. This probability distribution is updated at each time instant the image is captured and processed, the measurement update, and also in between these time instants, the time update. This process is called stochastic filtering.

From now on we will denote by $t$ - the time instant just before processing an image at time $t$, and by $t+$ the time instant just after processing an image at time $t$. We will also assume that $X_{t}$ is normally distributed, $X_{t} \sim N\left(\hat{X}_{t}, P_{t}\right)$, and therefore fully specified by its mean $\hat{X}_{t}$ and covariance $P_{t}$. The probability distribution updates are then identical to updates of the mean and covariance.

\footnotetext{
${ }^{1}$ Homogeneous coordinates of a point in $\mathbb{R}^{3}$ is a 4 -vector $p=(a x, a y, a z, a)^{t}$, where $x, y, z$ are the coordinates of the point in a common sense, and $a$ is an arbitrary nonzero real number. In our case $a$ is always equal to 1 .
} 


\subsection{Measurement update}

Line segments found in processed images are subjected to random perturbation due to imaging errors, presence of noise, etc. The observation vector $y_{t}$ obtained from an image at time $t$ can be also considered to be an outcome of the random variable $Y_{t}$. We may assume that

$$
Y_{t}=h\left(X_{t-}\right)+\xi_{t}, \quad \xi_{t} \sim N\left(0, R_{t}\right)
$$

where $h\left(X_{t-}\right)$ is an ideal projection of line segments onto the image plane using equations (1) and (2), and $\xi_{t}$ is a random perturbation having normal distribution with zero mean and covariance $R_{t}$.

Probability distribution of $X_{t+}$ after $Y_{t}=y_{t}$ has been observed can be expressed in terms of probability density functions (pdf-s) using Bayes rule

$$
f_{X_{t+}}(x)=f_{X_{t-} \mid Y_{t}}\left(x \mid y_{t}\right)=\frac{f_{X_{t-}, Y_{t}}\left(x, y_{t}\right)}{f_{Y_{t}}\left(y_{t}\right)}=\frac{f_{Y_{t} \mid X_{t-}}\left(y_{t} \mid x\right) f_{X_{t}-}(x)}{f_{Y_{t}}\left(y_{t}\right)} .
$$

The density $f_{X_{t-}}(x)$ is the density of $X$ before making the observation and from equation (5) it follows that the so-called likelihood function $f_{Y_{t} \mid X_{t-}}\left(y_{t} \mid x\right)$ is

$$
f_{Y_{t} \mid X_{t-}}\left(y_{t} \mid x\right)=f_{\xi_{t}}\left(y_{t}-h(x)\right) .
$$

Due to a fact that the likelihood function (7) is a nonlinear function of state and observation vectors, the updated distribution of $X_{t+}$ will not be normal. However, it may be approximated by a normal distribution obtained in the following way:

1. A maximum a posteriori (MAP) estimate of state $x$, that is $x=\hat{x}_{\text {map }}$ maximizing $f_{X_{t+}}\left(x \mid y_{t}\right)$ is computed:

$$
\hat{x}_{\text {map }}=\operatorname{argmax}\left\{f_{X_{t+}}\left(x \mid y_{t}\right)\right\}=\operatorname{argmax}\left\{f_{Y_{t} \mid X_{t-}}\left(y_{t} \mid x\right) f_{X_{t-}}(x)\right\} .
$$

2. The nonlinear function $h(x)$ is linearized at point $x=\hat{x}_{\text {map }}$, so that

$$
h(x) \approx h\left(\hat{x}_{\text {map }}\right)+\nabla_{h\left(\hat{x}_{\text {map }}\right)}\left(x-\hat{x}_{\text {map }}\right),
$$

where $\nabla_{h\left(\hat{x}_{\text {map }}\right)}$ is the Jacobian of $h\left(\hat{x}_{\text {map }}\right)$.

3. Approximation (9) is substituted into (7) and the Bayes rule is then evaluated. This leads to a normal a posteriori distribution $X_{t+} \sim N\left(\hat{x}_{\text {map }}, P_{t+}\right)$, with

$$
P_{t+}=\left[P_{t-}^{-1}+\nabla_{h\left(\hat{x}_{\text {map }}\right)}^{t} R_{t}^{-1} \nabla_{h\left(\hat{x}_{\text {map }}\right)}\right]^{-1} .
$$

From formula (10) it follows that by measurement update the uncertainty of the state is decreased. 


\subsection{Time update}

Between two observations, the state evolves in time in the following way:

$$
\begin{aligned}
l_{i,(t+\Delta t)-} & =l_{i, t+} \quad \text { for } i=1, \ldots, n \\
\theta_{p,(t+\Delta t)-} & =\theta_{c, t+} \\
\theta_{c,(t+\Delta t)-} & =2 \theta_{c, t+}-\theta_{p, t}+v_{t} .
\end{aligned}
$$

or

$$
X_{(t+\Delta t)-}=\Phi_{t+\Delta t, t} X_{t+}+\omega_{t}, \quad \omega \sim N\left(0, Q_{t}\right) .
$$

Equations (11a) represent the fact that the scene is static, equation (11c) states that the camera is moving smoothly, so that its position can be extrapolated linearly and $v_{t}$ is the random deviation of the camera position from an expected one.

Then the state transition in time is given by the following equations

$$
\begin{aligned}
\hat{X}_{(t+\Delta t)-} & =\Phi_{t+\Delta t, t} \hat{X}_{t+} \\
P_{(t+\Delta t)-} & =\Phi_{t+\Delta t, t} P_{t+} \Phi_{t+\Delta t, t}^{t}+Q_{t} .
\end{aligned}
$$

with $\hat{X}_{(t+\Delta t)-}$ and $P_{(t+\Delta t)-}$ being the mean and covariance of the updated probability distribution of $X$ at time $t+\Delta t$ before processing the next image. From (13) it follows that the uncertainty of the state (namely of the camera parameters) is increased.

\subsection{State initialization}

The a priori covariances of the unknown parameters are set to (some) large values. The uncertainty of the few initial camera positions is diminished by the measurement updates, when a known part of the scene is captured. When new lines enter the camera view, their processing is deferred until the next image, when the corresponding line pairs are formed. Initial line parameter estimates are set to values obtained by the triangulation, and the initial covariances are also set to large values. By processing subsequent images, the uncertainty of the parameters is diminishing.

\subsection{Tracking line segments}

Correspondence between line segments in subsequent images is provided by tracking 2-D line segments in the 2-D image plane. If the time difference between images from a smoothly moving camera is small, line movement on the 2-D image plane is smooth too. This can be used to predict the next position of the 2-D line segment by a Kalman filter (see also [4] and [5]). A state vector of a 2-D line segment contains information about the line segment's position, velocity and acceleration. The predictions of the line segment's position in the next image are made using an assumption that 2-D lines are moving with constant acceleration. An actually observed line segment is assigned to the predicted one, if the Mahanalobis distance between the predicted and the observed line is smaller than some threshold. 


\subsection{Efficiency of computations}

The most time consuming operations involved in stochastic filtering are the maximization problem (8), and the covariance update (13). The dimensions of the state vector, the observation vector and the covariance matrices might be very large, for instance for 100 lines the covariance $P_{t}$ has the dimension $612 \times$ 612.

To avoid computation of matrix inversions and square roots ${ }^{2}$, we have used a procedure updating directly a square root of the inverse of the covariance matrices [9]. Equation (10) is rewritten as

$$
S_{t+}^{-t} S_{t+}^{-1}=S_{t-}^{-t} S_{t-}^{-1}+\nabla_{h(\hat{x} \mathrm{map})}^{t} S_{R}^{-t} S_{R}^{-1} \nabla_{h\left(\hat{x}_{\mathrm{map}}\right)}
$$

where $P_{t+}^{-1}=S_{t+}^{-t} S_{t+}^{-1}, P_{t+}^{-1}=S_{t+}^{-t} S_{t+}^{-1}$, and $R_{t}^{-1}=S_{R}^{-t} S_{R}^{-1}$. One solution of (14) with respect to $S_{t+}^{-1}$ is

$$
\tilde{S}_{t+}^{-1}=\left(\begin{array}{c}
S_{t-}^{-1} \\
S_{R}^{-1} \nabla_{h\left(\hat{x}_{\text {map }}\right)}
\end{array}\right) .
$$

This solution does not have the same dimension as $S_{t-}^{-1}$, however, it can transformed by QR-decomposition into a form

$$
Q^{t} \tilde{S}_{t+}^{-1}=\left(\begin{array}{c}
S_{t+}^{-1} \\
0
\end{array}\right)
$$

$S_{t+}^{-1}$ is an upper triangular matrix and the requested solution of (14) has a proper dimension. By exploiting the structure of matrices $S_{t-}^{-1}$ and $S_{R}^{-1} \nabla_{h(\hat{x} \text { map })}$, QRdecomposition can be performed line by line in a time linear with the number of lines [2].

Solution of (14) is requested as well when maximizing (8) by iterations. The overall improvement of the computation time by avoiding matrix inverses, square roots and by exploiting the structure of matrices is more than 100 -fold!

\section{Implementation and results}

The 3-D scene reconstruction has been implemented in Matlab Numeric Computation and Visualization Software. The Matlab program processes the line images with an already established correspondence between lines in subsequent images. The processing is performed off line.

We have processed a sequence of images of the scene shown in figure 1. The camera was moving on a rail above the scene with a constant speed of $140 \mathrm{~mm}$ per image, and at the same time it was rotating around the axis with the speed of $2^{\circ}$ per image. The objects were at a distance of about $4 \mathrm{~m}$ from the camera. The image sequence with the extracted line segments is shown in figure 2 . The edges

${ }^{2}$ The square root of a matrix $P$ is is a matrix $S$ such that $P=S S^{t}$. If some matrix $S$ is a square root of $P$, then matrix $S Q$, where $Q$ is an orthogonal matrix, is also a square root of $P$. 


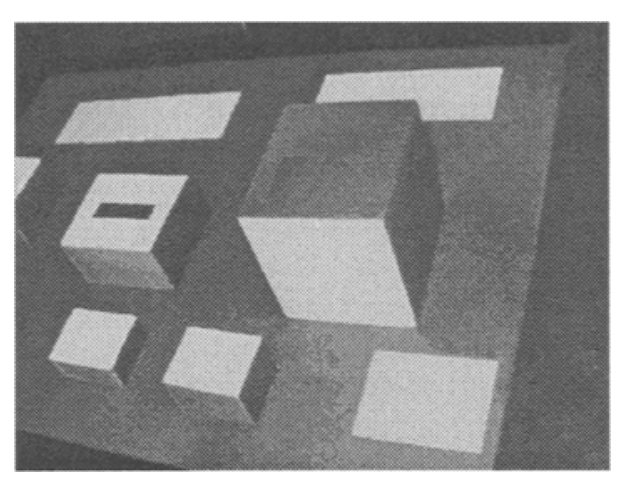

Fig. 1. Image of the scene.

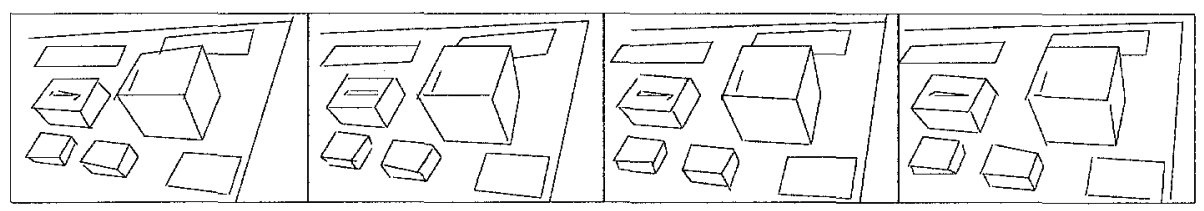

Fig. 2. Sequence of four images. Line segments were extracted from intensity images.

of the largest cube in the scene were given accurately and served as reference lines. The reconstructed 3-D lines are shown in figure 3 , together with the lines of the actual scene. The elements of the covariance matrix of the state were
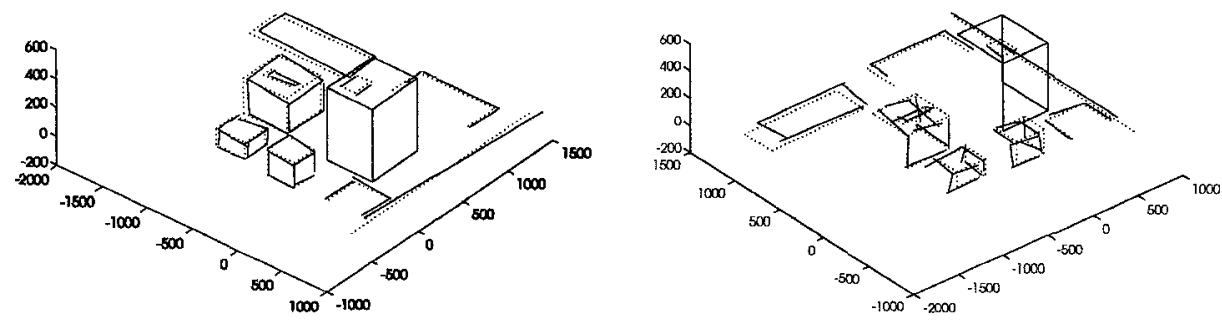

Fig. 3. The result of the $3-\mathrm{D}$ scene reconstruction depicted from two different viewpoints. Solid lines are the reconstructed lines, and dotted lines are the line segments of the actual scene.

decreasing rapidly, the standard deviations of line parameters are below $50 \mathrm{~mm}$ after processing four images. 


\section{Conclusions and future work}

In this paper the 3 -D scene reconstruction problem is viewed as a stochastic filtering problem, where the state is a parametric representation of the scene and the camera position, and images are the observations. At the moment, the scene is limited to a set of line segments. This technique can be extended to other different features, like circular arcs, etc., by parameterizing them and using an appropriate projection equation (equation (5)) in the measurement update.

Another question is, whether the assumptions made about the distribution of perturbations and the state are valid. If there are many noise sources that influence the random perturbations $\xi_{t}$ and $\omega_{t}$, then their distribution tends to be Gaussian. This leads to approximately Gaussian distribution of the state $X_{t}$ after the updates. An accurate identification of distributions of $\xi_{t}$ and $\omega_{t}$ is essential for the overall accuracy, and will be a topic of the future research.

We have proposed the algorithm reducing the computation time to time linear with the number of lines in the images. It should be possible to implement the system in real time. A future scenario is, that a person carrying the camera is scanning an industrial object and measures with the system its geometrical description. The system would be applicable in the CAD/CAM area.

\section{References}

1. N. Ayache and F. Lustman. Trinocular stereo vision for robotics. IEEE Trans. Patt. Anal. Machine Intell., 13:72-85, 1991.

2. M. G. Cox. The least squares solution of overdetermined linear equations having band or augmented band structure. IMA J. of Num. Analysis, (1):3-22, 1981.

3. J. Crowley and P. Stelmaszyk. Measurement and integration of $3 \mathrm{D}$ structures by tracking edge lines. In Proc. 1st Eur. Conf. Comp. Vision, pp. 269-280, Apr. 1990.

4. J. Crowley, P. Stelmaszyk, and C. Discours. Measuring image flow by tracking edge-lines. In ICCV 88: 2nd Int. Conf. on Comp. Vision, pp. 658-664, Dec. 1988.

5. R. Deriche and O. Faugeras. Tracking line segments. Image and Vision Computing, 8(4):261-270, 1990 .

6. O. Faugeras et al. The depth and motion analysis machine. IEEE Trans. Pattern Anal. Machine Intell., 6:353-385, Aug. 1992.

7. L. Hong and D. Brzakovic. 3D scene reconstruction from noisy image sequences using data fusion. Control Engineering Practice, 2(5):825-831, 1994.

8. L. Hong and D. Brzakovic. Distributed algorithm for multi-sensor integration applied to 3D scene reconstruction. 1994.

9. P. S. Maybeck. Stochastic Models, Estimation and Control, volume 1. Academic Press, 1979.

10. Z. Zhang and O. Faugeras. Building a 3D world model with a mobile robot: $3 \mathrm{D}$ line segment representation and integration. In Proc. 10th Int. Conf. Pattern Recognition, pp. 38-42, June 1990.

11. Z. Zhang and O. Faugeras. Estimation of displacements from two 3D frames obtained from stereo. IEEE Trans. Patt. Anal. Mach. Int., 14(12):1141-1156, 1992. 